\title{
Mating-Induced Trade-Offs upon Egg Production versus Fertilization and Offspring's Survival in a Sawfly with Facultative Parthenogenesis
}

\author{
Hong Yu ${ }^{1}$, Min-Rui Shi ${ }^{1}$, Jin Xu ${ }^{1,2, *}$, Peng Chen ${ }^{3}$ and Jian-Hong Liu ${ }^{1, *}$ \\ 1 Yunnan Academy of Biodiversity, Southwest Forestry University, Kunming 650224, China; \\ yuhong1652@126.com (H.Y.); smr1997by@163.com (M.-R.S.) \\ 2 Key Laboratory for Forest Resources Conservation and Utilization in the Southwest Mountains of China, \\ Ministry of Education, Southwest Forestry University, Kunming 650224, China \\ 3 Yunnan Academy of Forestry and Grassland, Kunming 650201, China; pengchenn@126.com \\ * Correspondence: xujin2798@126.com (J.X.); jianhongliu6@163.com (J.-H.L.)
}

check for updates

Citation: Yu, H.; Shi, M.-R.; Xu, J.; Chen, P.; Liu, J.-H. Mating-Induced Trade-Offs upon Egg Production versus Fertilization and Offspring's Survival in a Sawfly with Facultative Parthenogenesis. Insects 2021, 12, 693. https://doi.org/10.3390/ insects12080693

Academic Editors: Clint Kelly and Geoffrey M. Attardo

Received: 27 May 2021

Accepted: 29 July 2021

Published: 2 August 2021

Publisher's Note: MDPI stays neutral with regard to jurisdictional claims in published maps and institutional affiliations.

Copyright: (c) 2021 by the authors. Licensee MDPI, Basel, Switzerland. This article is an open access article distributed under the terms and conditions of the Creative Commons Attribution (CC BY) license (https:/ / creativecommons.org/licenses/by/ $4.0 /)$.
Simple Summary: Study of mating-induced trade-offs between reproduction and survival is conducive to provide evolutionary insights into reproductive strategies and aging. Using RNA sequencing and bioinformatics, we found that mating induced changes of genes and pathways related to reproduction and survival in females of a pine sawfly. Mating induced substantial downregulation on genes associated to immunity, stress response, and longevity. However, mating induced divergent reproductive response, with downregulation on genes related to egg production while upregulation on genes related to egg fertilization. Considering the nature of limited resources in adults, low fecundity and egg protection behavior in this sawfly, we suggest that mating triggers trade-offs between reproduction and survival in this insect and females of this species have evolved specific strategies to adapt to the living conditions, e.g., restrict whole fecundity to ensure higher fertilization and offspring's survival.

Abstract: Investigation of mating-induced trade-offs between reproduction and survival is conducive to provide evolutionary insights into reproductive strategies and aging. Here, we used RNAseq and bioinformatics to reveal mating-induced changes of genes and pathways related to reproduction and survival in female Cephalcia chuxiongica, a pine defoliator with facultative parthenogenesis and long larval dormancy. Results showed that mating induced substantial downregulation on genes and pathways associated to immunity, stress response, and longevity. However, mating induced divergent reproductive response, with downregulation on genes and pathways related to egg production while upregulation on genes and pathways related to egg fertilization. Considering the nature of limited resources in adults, low fecundity, and egg protection behavior in C. chuxiongica, we suggest that mating triggers trade-offs between reproduction and survival in this insect and females of this species may have evolved specific strategies to adapt to the environmental and hosts' conditions, e.g., restrict whole fecundity to ensure higher fertilization and offspring's survival. Moreover, mating induced significant responses on genes and pathways that play important roles in vertebrate reproduction while their function in insects are unclear, such as the progesterone-mediated oocyte maturation pathway; the significant regulation after mating suggests that their function may be evolutionarily conserved in animal kingdom.

Keywords: Cephalcia chuxiongica; mating-responsive genes; egg production; egg fertilization; offspring's survival; somatic maintenance

\section{Introduction}

Trade-offs between reproduction and survivorship is the central theme in evolutionary biology of senescence. Negative relationships between reproduction and survivorship are 
often observed in organisms, in which increased fecundity may cause costs on somatic maintenance (such as immunity and stress responses) and longevity [1-4]. The resources allocation model suggests that the trade-offs between reproduction and survival is likely due to alternative allocation of limiting energetic resources because both reproduction and soma maintenance are energetically costly $[1,5]$. Genetic model suggests that there are antagonistic alleles that may promote negative genetic correlation between reproduction and survival, i.e., alleles may promote fecundity at the expense of somatic maintenance $[2,6]$. These two models are not mutually exclusive [2,7].

In insects, the endocrine network plays vital roles in reproduction and maintenance regulation, which mainly involves insulin-like/IGF-1 signaling (IIS), juvenile hormone (JH), 20-Hydroxyecdysone (20E), yolk precursor vitellogenin (Vg), and yolk proteins (YPs) [2]. $\mathrm{JH}$ and IIS are important mediators of egg maturation; improved JH and IIS levels promote the expression of $\mathrm{Vg}$ and YPs and the uptaking of $\mathrm{Vg}$ and YPs into oocytes [1]. However, elevated JH and IIS may inhibit immune responses, such as by reducing phenoloxidase activity and the production of antimicrobial peptides $[1,8]$. In contrast, $20 \mathrm{E}$ may promote immune responses, such as by upregulating the expression of antimicrobial peptide genes in Drosophila melanogaster [8,9]. Therefore, although the mechanism remains poorly understood, such opposite effects of different hormones seem to be intimately involved in the trade-offs between reproduction and survivorship [1,2].

Female insects are likely to face a limited protein supply during reproductive period because many of them do not feed on a protein source as adults while elevated egg production process requires higher protein supplies at this stage [10-12]. Female insects thus may have evolved behavioral and physiological strategies to allocate limited resources optimally between reproduction and survival [1,13]. Mating is a key switch for sexual reproduction in insects, which incurs major changes in the physiology and behavior in females [14]. Previous studies have found that mating did positively affect female reproduction and negatively affect female immune activities in some insects $[1,5,15]$. However, some studies also found that there is no difference in immune responses between mated and virgin insect females and in some insects mating even upregulates female immune responses [5]. These diverse findings may be due to the differences in measurement methods and immune indicators and the differences in species and mating systems. For example, males may directly suppress female immunity, thereby promoting sperm storage and egg fertilization by their sperms in female reproductive tracts [16]. However, mated females may need to have higher post-mating immune activity to provide defense against mating transferred infections, particularly in polygamous mating systems [5,17]. The heat shock response is essential for proteostasis and cellular health [4]. Similarly, studies on reproduction-induced heat shock response also found inconsistent results. In C. elegans, the heat shock response was inhibited at the onset of reproduction, probably due to competing requirements of the germline and soma [4]. In honey bees, however, increased reproduction did not cause loss of heat shock response in the reproductive queen [3]. Therefore, honey bee queens may possess an atypical uncoupling of the reproduction-maintenance trade-off, in which stress response may be maintained during reproduction [3]. Therefore, study on the trade-offs between reproduction and survival in different mating systems by using methods that can more comprehensively examine various indicators (such as high-throughput sequencing and bioinformatics) will provide deeper insights in this field.

Recent progress on high-throughput sequencing and bioinformatics has revolutionized our understanding of life and organism. Previous transcriptome analysis in a number of insect species have shown that mating can induce expression changes in genes related to reproduction, immunity, stress response, and longevity [18-20]. A resent transcriptomebased study in the sweet potato whitefly females found 434 deferentially expressed genes (DEGs) between mated and unmated groups, with many of them encoding binding-related proteins and genes associated with lifespan [20]. Another study in Spodoptera litura female moths showed a divergent response in DEGs in relation to reproduction and immunity [15]. These results suggest that trade-offs on reproduction versus survival were induced by 
mating in some insects. In the present study, we studied genes and pathways related to reproduction and survival (immunity, stress response, and longevity) and mating-induced regulation in females of a pine sawfly, Cephalcia chuxiongica. This insect has a facultative parthenogenesis reproductive system and a long (19 months) larval dormancy stage [21]. Study of mating-induced regulation on reproduction and survivorship in such a special insect species is expected to achieve some new enlightenment on the evolution of reproductive strategies and aging.

The sawfly Cephalcia chuxiongica Xiao (Hymenoptera: Pamphiliidae) is a serious pest of pines in China [22,23]. Almost fifty Cephalcia species have been found all over the world, with most of them being important forest pests [24,25]. Cephalcia chuxiongica larvae feed on pine needles and pupate in the soil under the trees. The life cycle of this species is long (about 22 months) due to a 19-month larval diapause in the soil [22]. Similar to many other species from Hymenoptera, C. chuxiongica females perform sexual reproduction under normal conditions, while in the absence of males, they can perform parthenogenesis [22]. The male and female adults of $C$. chuxiongica can mate a few hours after eclosion; unmated females (virgin) may start to lay unfertilized eggs through parthenogenesis a few days ( $>3 \mathrm{~d}$ ) after eclosion [21]. Sawflies do most of their feeding during the larval stage, and adults of some species may feed on pollen and nectar [26]. So far, there is no report on adult feeding habits in C. chuxiongica; adults even did not feed on the provided honey solution, suggesting that this species do not feed during the adult stage [21].

\section{Materials and Methods}

\subsection{Sample Collection}

Cephalcia chuxiongica adults were collected in August 2019 under infested pine trees in the Pinus yunnanensis forest, located on a small mountain (25.600166N, 103.439364E) near Xundian town in Yunnan Province, China. Cephalcia chuxiongica pupate and eclose underground during July to Sept. Eclosed adults crawl out from the soil for reproduction in the forest. To confirm adults virginity and age, newly eclosed adults were directly dug out from the soil below the pine trees in the morning [27]. Males and females were sexed according to morphological traits [23] and reared separately on the pine trees (on the pine needles covered by nylon mesh bag [30 cm diameter, $40 \mathrm{~cm}$ long] to avoid insect escape). As mentioned above, $C$. chuxiongica adults can mate soon after emergence and mating mostly happen at noon. Therefore, mating was allowed in the same day after collection during 12:00-14:00 by pairing males and females on the pine needles covered by bags with one pair per bag. Mating events (two insects engaged at the tip of the abdomen; mating duration is about $20 \mathrm{~min}$ ) [22] were recorded. Males were removed after mating and the mated females were then individually reared in the same bag and their abdomens were sampled at $1 \mathrm{~h}, 6 \mathrm{~h}$, and $24 \mathrm{~h}$ after mating. Abdomens from fifteen females were used as a replicate, and three replicates were used for each sampling time point. Virgin females at the same age as mated individuals were used as controls. All samples were placed in liquid nitrogen for immediate freezing after sampling and stored at $-80{ }^{\circ} \mathrm{C}$. One replicate from Virgin- $1 \mathrm{~h}$ and one replicate from Virgin- $6 \mathrm{~h}$ were discarded due to lower sequencing quality (see below) and thus finally, two replicates for Virgin- $1 \mathrm{~h}$ and Virgin- $6 \mathrm{~h}$, and three replicates for other treatments (Virgin-24 h, Mated-1 h, Mated-6 h and Mated-24 h) were used for analysis.

\subsection{Library Preparation and Sequencing}

Total RNA was extracted from samples using Trizol reagent (Invitrogen Inc., Calsbad, CA, USA) and then was treated with the RNase-free DNase I to eliminate genomic DNA. The purity and concentration of RNA was assessed by using Qubit RNA Assay Kit (Life Technologies Inc., Grand Island, NY, USA) and the NanoPhotemeter spectrophotometer (Implen Inc., Westlake Village, CA, USA). The RNA integrity was checked by the Agilent Bioanalyzer 2100 system (Agilent Technologies Inc., Palo Alto, CA, USA). One microgram RNA per sample was used for the preparation of the sequencing libraries by using NEBnext 
Ultra RNA Library Prep Kit for Illumina (New England BioLabs Inc., San Diego, MA, USA) following the manufacturer's instructions and index codes were added to attribute sequences to each sample. The quality of each sample library was assessed using the Agilent Bioanalyzer 2100 system. Ultimately the library per sample was sequenced by Illumina Hiseq4000 platform (Majorbio Biotechnology Inc., Shanghai, China) and the paired-end reads were generated.

\subsection{Quality Control and Assembly}

The raw reads obtained were initially processed by trimming the adapter and low quality reads to produce clean reads. The clean reads were assembled using the Trinity software (version 2.5.1) to generate transcripts [28]. Then transcript analysis was performed to remove redundancies with TGICL software (version 2.1) and acquire unigenes without redundancy [29].

\subsection{Differential Expression Analysis}

Gene expression levels were determined as transcripts per million (TPM). The differential expression analysis between samples was performed using the edgeR $\mathrm{R}$ package (3.0.8). $p$-value was adjusted using $q$-value [30]. $q<0.05$ and $\mid \log 2$ (foldchange) $\mid>1$ was set as the threshold for significantly differential expression.

\subsection{Functional Annotation and Enrichment Analysis of Differentially Expressed Genes (DEGs)}

Using the BLAST [31] software, the DEGs were compared with NCBI non-redundant protein (NR), Swiss-Prot protein database (Swiss-Prot), Gene Ontology (GO), KEGG Orthology (KO), Cluster of Orthologous Groups (COG), and Pfam databases to obtain annotation information about the DEGs. GO enrichment analysis of DEGs was implemented by using the GOSeq program and KEGG enrichment was performed using the KOBAS software. GO terms and KEGG pathways with $q<0.05$ were significantly enriched in DEGs.

\subsection{Validation by $q R T-P C R$}

To verify the accuracy of the RNAseq data, 24 DEGs were used for qRT-PCR and the $\beta$-Tubulin (GeneBank ID: MZ028603) was used as a reference gene. Total RNA was extracted from above samples by using RNAiso plus (TaKaRa Inc., Dalian, China) and cDNA was synthesized using PrimeScript RT reagent Kit (Takara Inc., Dalian, China). The PCR was performed by QuantStudio 7 Flex (Thermo Fisher Scientific Inc., Waltham, MA, USA) with gene specific primers (Table S1) and the following program: $95{ }^{\circ} \mathrm{C}$ for $30 \mathrm{~s}$, followed by 40 cycles of $95^{\circ} \mathrm{C}$ for $5 \mathrm{~s}, 60^{\circ} \mathrm{C}$ for $30 \mathrm{~s}$, and dissociation. The $2^{-\Delta \Delta \mathrm{CT}}$ method [32] was used to calculate the relative expression. Differences of gene expression levels between treatments were analyzed by ANOVA using SPSS 25.0. The rejection level was set at $\alpha<0.05$.

\section{Results}

\subsection{Sequencing and Assembly}

By RNAseq using Illumina HiSeq4000 platform, $~ 55,000,000$ clean reads were obtained from each of the 16 sequenced libraries (Table S2). The percentages of Q20 and Q30 of all samples' clean reads ranged from $97.76 \%$ to $98.21 \%$ and from $94.10 \%$ to $95.21 \%$, respectively. The biological replicates were highly correlated (Table S3), which affirmed the reproducibility of the RNASeq technology and biological replicates. The transcriptome raw reads were deposited to the NCBI SRA database (accession no.: SRR13991392 SRR13991407).

\subsection{Outline of Mating-Induced Transcriptional Changes}

There are 149, 320, and 1068 DEGs within Mated-1 h vs. Virgin-1 h (M1 h-vs.-V1 h), M6 h-vs.-V6 h and M24 h-vs.-V24 h groups, respectively (Figure 1; Tables S4-S6). There were 89 DEGs shared between M1 h-vs.-V1 h and M1 h-vs.-V1 h, 4 DEGs shared between M1 h-vs.-V1 h and M24 h-vs.-V24 h, and 36 DEGs shared between M6 h-vs.-V6 h and M24 
h-vs.-V24 h, while only one DEG (DN61334_c1_g3, hemolymph glycoprotein, downregulated) was shared by the three groups (M1 h-vs.-V1 h, M6 h-vs.-V6 h and M24 h-vs.-V24 h) (Figure 2; Table S7).
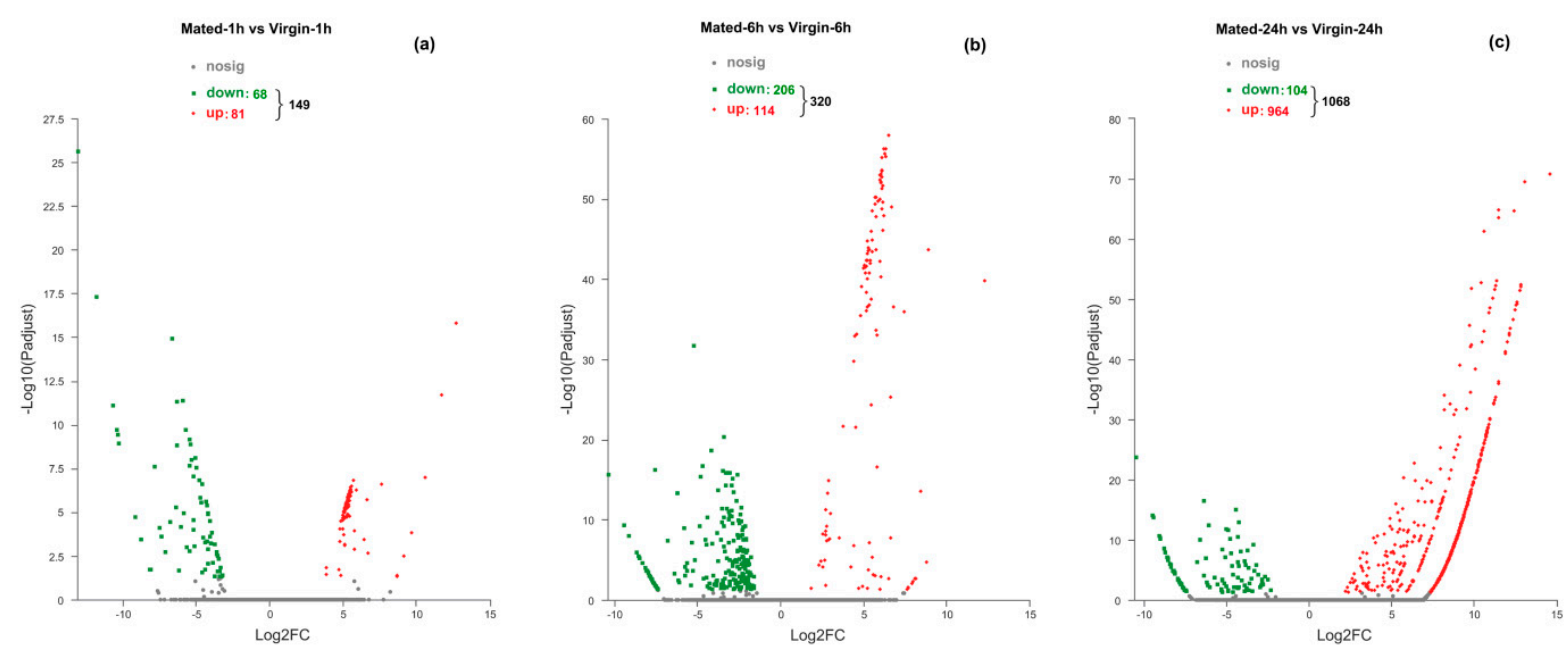

Figure 1. Volcano plots of the DEGs in Mated-1 h vs. Virgin-1 h group (a), Mated-6 h vs. Virgin-6 h group (b), and Mated-24 h vs. Virgin-24 h group (c). Genes with significant differential expression were indicated by red dots (upregulated) and green dots (downregulated). Genes with no significant differential expression were represented by grey dots.

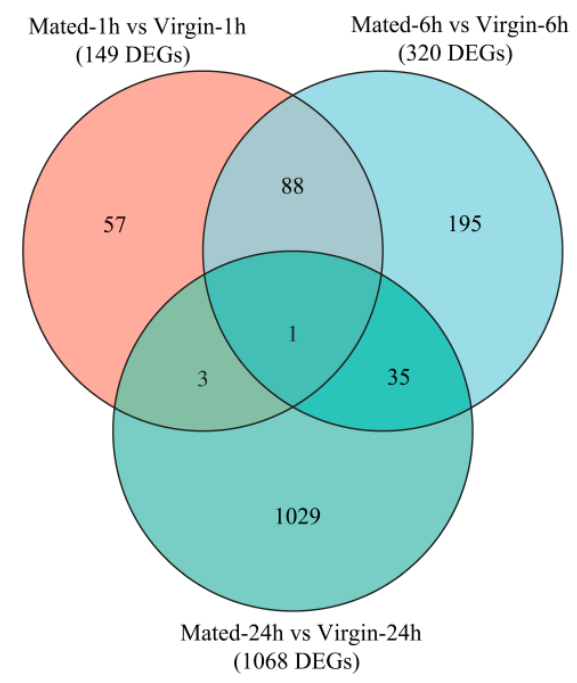

Figure 2. The Venn diagram of DEGs. The overlapping circles represented common DEGs among all combinations.

To better understand their functions, all these DEGs were annotated based on NR, Swiss-Prot, GO, KO, COG, and Pfam databases (Tables S4-S6) and then were submitted for GO (Table S8) and KEGG (Table S9) enrichment analysis. Most of these DEGs $(1235 / 1537=80.4 \%)$ were successfully mapped to at least one of these databases (Tables S4-S6). A total of 309 GO terms were significantly enriched in these DEGs, with most of them (59.5\%) being assigned to biological process (BP) terms, and then $17.2 \%$ and $23.3 \%$ being assigned to cellular component (CC) terms and molecular function (MF) terms, respectively (Figures 3a and 4; Table S8). Moreover, there are 51 KEGG pathways that were significantly enriched in these DEGs, which were assigned to six of Level I Categories (Figures $3 b$ and 5; Table S9). 


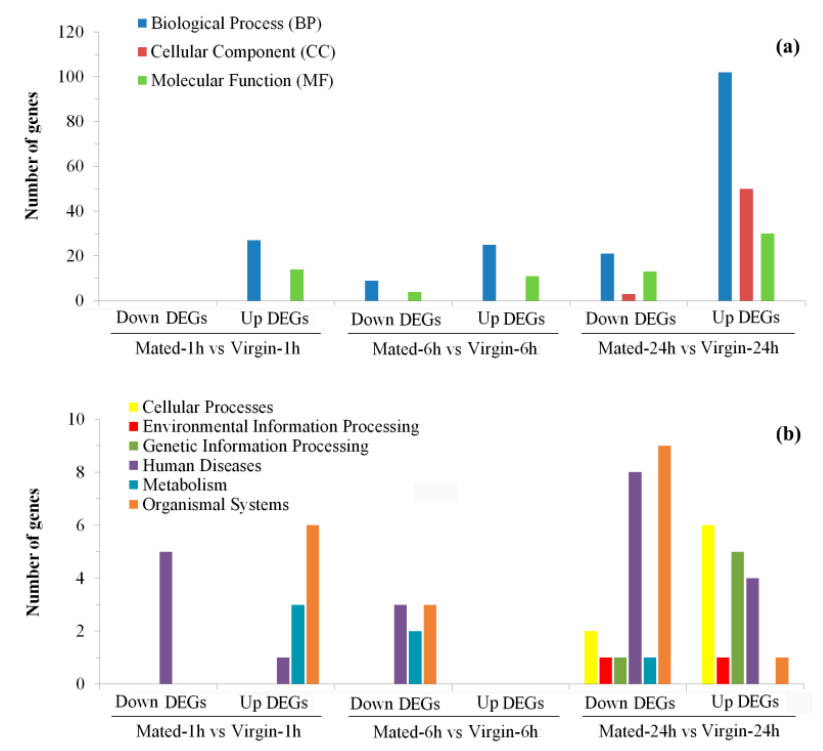

Figure 3. Summary of GO (a) and KEGG (b) enrichment analysis of DEGs in different groups.
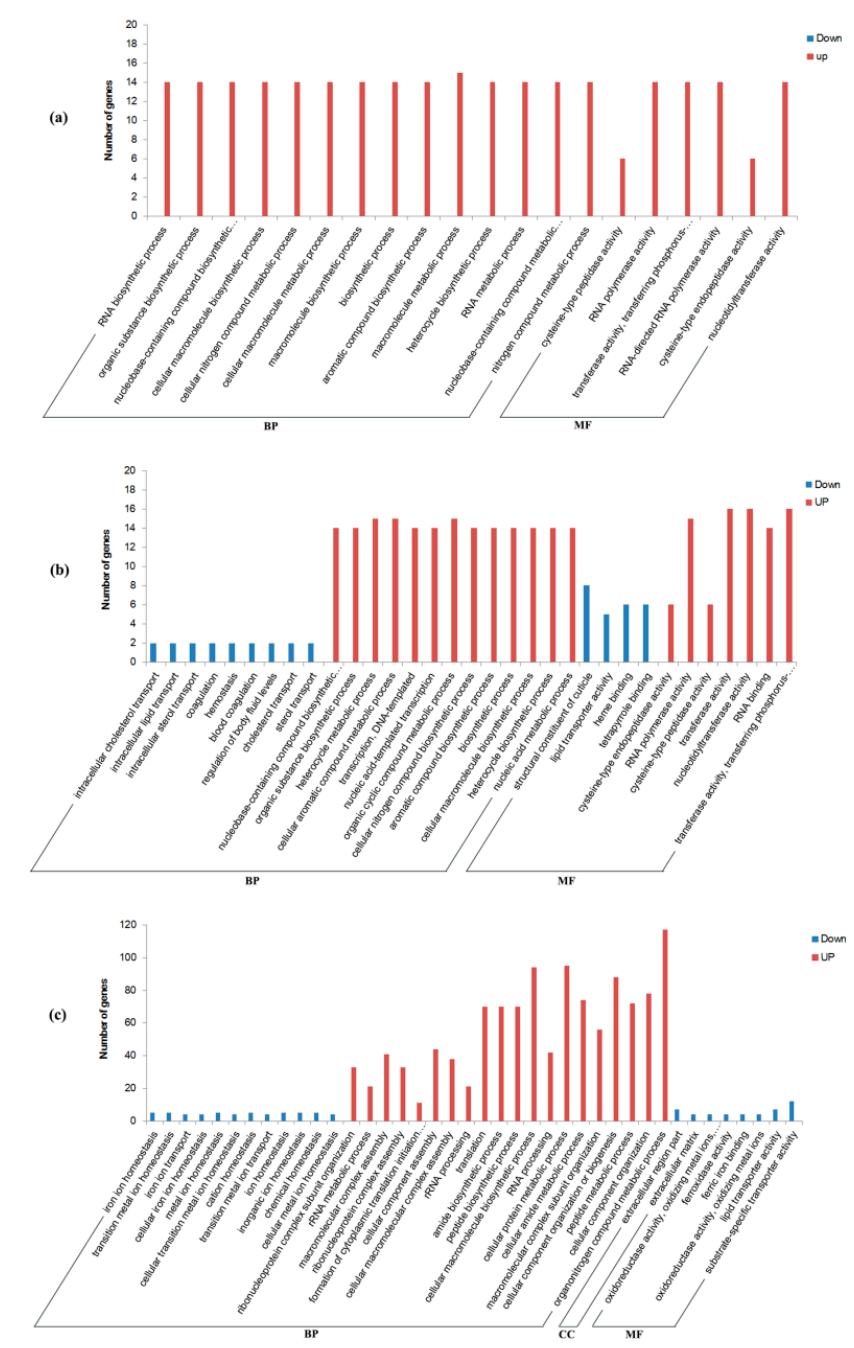

Figure 4. GO enrichment of DEGs in Mated-1 h vs. Virgin-1 h group (a), Mated-6 h vs. Virgin-6 h group (b), and Mated-24 h vs. Virgin-24 h group (c). The function of DEGs was divided into three parts: BP (biological process), CC (cell composition), and MF (molecular function). The red bars indicate upregulated DEGs and blue bars indicate downregulated DEGs. The top 20 terms each from upregulated DEGs and downregulated DEGs of each group are shown. 

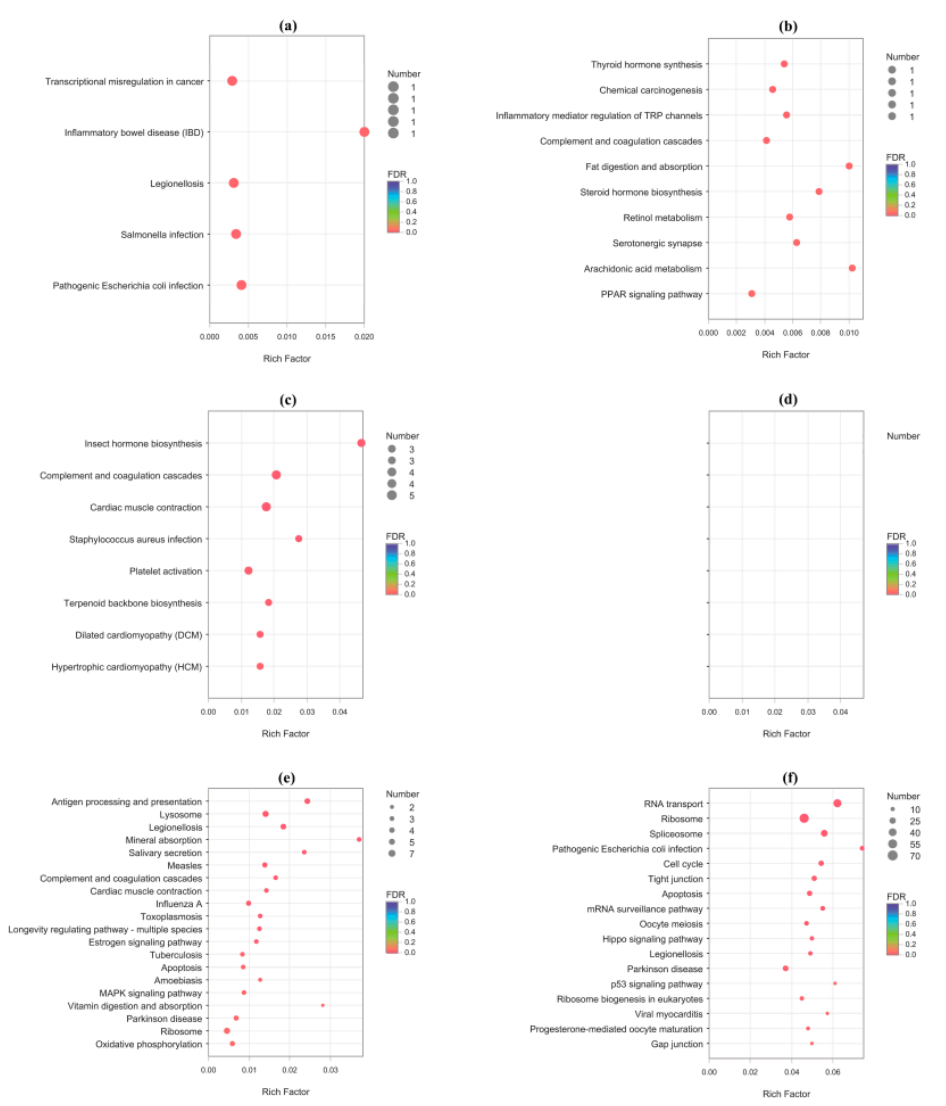

Figure 5. KEGG pathway enrichment of DEGs in Mated-1 h vs. Virgin-1 h group ((a) upregulated DEGs; (b) downregulated DEGs), Mated-6 h vs. Virgin-6 h group ((c) upregulated DEGs; (d) downregulated DEGs, no DEGs enriched), and Mated-24 h vs. Virgin-24 h group ((e) upregulated DEGs; (f) downregulated DEGs). The size of the dot indicates the number of DEGs in this pathway, and the color of the dot corresponds to different q-value ranges. The top 20 pathways each from upregulated DEGs and downregulated DEGs of each group are shown.

Mating-induced regulation on genes and pathways related to reproduction and survivorship were then studied in detail as follows based on the above annotation and enrichment analysis.

\subsection{Transcriptional Changes at $1 \mathrm{~h}$ Post-Mating}

At $1 \mathrm{~h}$ after mating, 68 genes were downregulated and 81 genes were upregulated in mated females compared to virgin females (Figure 1a). The log2FoldChange (LC) value of DEGs varied from -13.04 to 12.74 (Table S4).

Two reproduction-related genes were found within these DEGs, with one encoding follicle cell protein (downregulated) and one encoding cytochrome P450 (upregulated) (Table 1). KEGG enrichment analysis of the 149 DEGs found one reproductive related pathway, the steroid hormone biosynthesis pathway, which enriched to one upregulated DEG (Table 2).

Two immunity related genes were found within these DEGs (Table 3). Both of them encode antimicrobial peptides and both were downregulated in mated females compared to virgin ones. Six immunity related pathways were enriched based on the 149 DEGs, with four pathways enriched to downregulated DEGs and two pathways enriched to upregulated DEGs (Table 4).

No longevity and heat shock-related genes and pathways were found within these DEGs of M1 h-vs.-V1 h. 
Table 1. Mating-induced expression changes in genes related to reproduction in C. chuxiongica females.

\begin{tabular}{|c|c|c|c|c|c|}
\hline GeneID & \multicolumn{2}{|c|}{ log2FoldChangePadj } & Annotation & Function & Reference \\
\hline \multicolumn{6}{|c|}{ Mated-1 h vs. Virgin-1 h } \\
\hline DN64818_c0_g6 & -3.51325 & 0.02376 & Follicle cell protein $3 \mathrm{C}$ & $\begin{array}{l}\text { Encodes a major protein component of the } \\
\text { vitelline membrane. }\end{array}$ & [33] \\
\hline DN64030_c0_g1 & 9.66278 & 0.00015 & $\begin{array}{l}\text { CYP2C (Cytochrome P450 } \\
\text { family) }\end{array}$ & $\begin{array}{c}\text { Detoxification and the biosynthesis of } \\
\text { hormones. }\end{array}$ & [34] \\
\hline \multicolumn{6}{|c|}{ Mated-6 h vs. Virgin-6 h } \\
\hline DN65980_c5_g3 & -9.14964 & $1.05 \times 10^{-8}$ & Vitellogenin-2 & \multirow{5}{*}{$\begin{array}{l}\text { Plays vital role in oocytes and embryo } \\
\text { development in insects. }\end{array}$} & \multirow{5}{*}[35,36]{} \\
\hline N55390_c2_g3 & -7.75002 & 0.00289 & Vitellogenin-A2-like & & \\
\hline N52188_c2_g1 & -6.37534 & 0.00062 & Vitellogenin-A2-like & & \\
\hline DN71364_ct_ol & -2.39804 & $2.43 \times 10^{-11}$ & Vitellogenin-like & & \\
\hline DN59614_c4_g1 & -2.34561 & $1.55 \times 10^{-10}$ & Vitellogenin-like & & \\
\hline DN78694_c0_g1 & -3.45314 & 0.03932 & & \multirow{2}{*}{$\begin{array}{l}\text { Protect the labile hormone molecules from } \\
\text { degradation by esterase. }\end{array}$} & \multirow[b]{2}{*}[37,38]{} \\
\hline DN63882_c0_g1 & -2.75932 & $\begin{array}{c}0.00064 \\
104 \times 10^{-8}\end{array}$ & $\begin{array}{c}\text { Haemolymph juvenile hormone } \\
\text { binding protein }\end{array}$ & & \\
\hline $\begin{array}{l}\text { DN65663_c1_g1 } \\
\text { DN65191_c2_g1 }\end{array}$ & $\begin{array}{l}-2.48229 \\
-2.34190\end{array}$ & $\begin{array}{c}1.04 \times 10^{-0} \\
0.02528\end{array}$ & Protein takeout-like & \multirow[b]{2}{*}{$\begin{array}{l}\text { Takeout is part of a large gene family found } \\
\text { throughout insects with roles in metabolism, } \\
\text { circadian behavior, aging, and male } \\
\text { courtship behavior. }\end{array}$} & \multirow[b]{2}{*}{ [39] } \\
\hline DN69612_c3_g1 & -1.58633 & 0.03611 & Protein takeout-like & & \\
\hline$D N 66978 \_c 2 \_g 1$ & -2.71257 & 0.00840 & UDP-glucosyltransferase & \multirow{3}{*}{$\begin{array}{l}\text { Positive modulator of fecundity. } \\
\text { Some members of matrix metalloproteinase } \\
\text { may play roles in corpus luteum formation, } \\
\text { follicular development, and ovulation. }\end{array}$} & [40] \\
\hline DN71428_c0_g1 & -8.16014 & $9.90 \times 10^{-5}$ & Matrix metalloproteinase- 16 & & {$[41]$} \\
\hline DN69614_c4_g4 & -2.86011 & 0.04696 & CYP4 (Cytochrome P450 family) & & \multirow{4}{*}[34]{} \\
\hline DN52313_c1_g5 & -2.81364 & $3.33 \times 10^{-14}$ & CYP4 (Cytochrome P450 family) & \multirow{3}{*}{$\begin{array}{c}\text { Detoxification and the biosynthesis of } \\
\text { hormones. }\end{array}$} & \\
\hline DN69106_c0 91 & -2.15773 & 0.00170 & CYP4 (Cytochrome P450 family) & & \\
\hline DN53829_c6_g2 & -2.00810 & 0.01412 & CYP4 (Cytochrome P450 family) & & \\
\hline \multicolumn{6}{|c|}{ Mated-24 h vs. Virgin-24 h } \\
\hline DN65980_c5_g3 & -9.44195 & $1.71 \times 10^{-14}$ & Vitellogenin-2 & \multirow{9}{*}{$\begin{array}{l}\text { Plays vital role in oocytes and embryo } \\
\text { development in insects. }\end{array}$} & \multirow{9}{*}[35,36]{} \\
\hline DN71047_c1_g1 & -8.99780 & $8.17 \times 10^{-11}$ & Vitellogenin-A2-like & & \\
\hline DN55390_c2_g3 & -8.55112 & $1.39 \times 10^{-7}$ & Vitellogenin-A2-like & & \\
\hline DN52188_c2_g1 & -8.51720 & $2.32 \times 10^{-7}$ & Vitellogenin-A2-like & & \\
\hline DN49694_c0_g1 & -2.51669 & 0.00038 & Vitellogenin-like & & \\
\hline DN59614_c4_g1 & -2.30309 & 0.02439 & Vitellogenin-like & & \\
\hline DN71364_c1_g1 & -2.31618 & 0.02594 & Vitellogenin-like & & \\
\hline DN54912_c7_g1 & 9.02029 & $1.51 \times 10^{-11}$ & Vitellogenin receptor & & \\
\hline$D N 58744 \_c 7 \_g 1$ & 9.28253 & $8.58 \times 10^{-14}$ & $\begin{array}{c}\text { Zona pellucida sperm-binding } \\
\text { protein }(Z P) \text { 1-like }\end{array}$ & & \\
\hline DN32813_c0_g1 & 9.32544 & $3.72 \times 10^{-14}$ & $\mathrm{Zp1}$ & \multirow{23}{*}{$\begin{array}{l}\text { The zona pellucida is a glycoprotein layer } \\
\text { surrounding the plasma membrane of } \\
\text { oocytes. It is a vital constitutive part of the } \\
\text { oocyte and functions in primary binding and } \\
\text { induction of the sperm acrosome reaction. }\end{array}$} & \multirow{23}{*}{ [42-44] } \\
\hline DN67043_c1_g1 & 11.27687 & $2.07 \times 10^{-52}$ & $\mathrm{Zp1}$ & & \\
\hline$D N 44634 \_c 0 \_g 2$ & 10.29005 & $2.97 \times 10^{-23}$ & $\mathrm{Zp2}$ & & \\
\hline DN93387_c0_g1 & 7.70158 & 0.002245 & $\mathrm{Zp3}$ & & \\
\hline DN50223_c1_g2 & 8.04870 & $5.28 \times 10^{-5}$ & $\mathrm{Zp3}$ & & \\
\hline$D N 12342 \_c 0 \_g 1$ & 8.89036 & $1.66 \times 10^{-10}$ & $\mathrm{Zp3}$ & & \\
\hline DN63118_c0_g5 & 9.43726 & $3.94 \times 10^{-15}$ & $\mathrm{Zp3}$ & & \\
\hline DN42788_c0_g1 & 9.81002 & $1.13 \times 10^{-18}$ & $\mathrm{Zp3}$ & & \\
\hline DN44618_c0_g1 & 9.87890 & $1.89 \times 10^{-52}$ & $\mathrm{Zp3}$ & & \\
\hline DN43523_c0_g1 & 10.0620 & $5.22 \times 10^{-21}$ & $\mathrm{Zp3}$ & & \\
\hline DN22329_c0_g1 & 10.18637 & $3.23 \times 10^{-22}$ & $\mathrm{Zp3}$ & & \\
\hline DN47551_c0_g1 & 10.48415 & $1.26 \times 10^{-43}$ & Zp3 & & \\
\hline$D N 48592 \_c 0 \_g 2$ & 11.49147 & $9.56 \times 10^{-37}$ & $\mathrm{Zp3}$ & & \\
\hline DN63118_c0_g2 & 11.49933 & $2.70 \times 10^{-64}$ & $\mathrm{Zp3}$ & & \\
\hline$D N 47452 \_c 0 \_g 3$ & 11.88468 & $1.13 \times 10^{-41}$ & $\mathrm{Zp3}$ & & \\
\hline DN49748_c0_g1 & 12.50758 & $6.61 \times 10^{-49}$ & $\mathrm{Zp3}$ & & \\
\hline DN100350_c0_g1 & 12.60210 & $5.74 \times 10^{-50}$ & $\mathrm{Zp3}$ & & \\
\hline DN63118_c0_g1 & 13.09691 & $3.27 \times 10^{-70}$ & $\mathrm{Zp3}$ & & \\
\hline DN67043_c2_g1 & 7.96939 & $4.36 \times 10^{-26}$ & $\mathrm{Zp4}$ & & \\
\hline DN67043_c1_g2 & 8.81695 & $6.03 \times 10^{-10}$ & $\mathrm{Zp4}$ & & \\
\hline DN72297_c0_g1 & 9.81754 & $9.66 \times 10^{-19}$ & $\mathrm{Zp4}$ & & \\
\hline DN67043_c2_g3 & 10.63913 & $5.50 \times 10^{-62}$ & $\mathrm{Zp4}$ & & \\
\hline DN67258_c0_g10 & 10.78996 & $1.26 \times 10^{-28}$ & $\mathrm{Zp4}$ & & \\
\hline
\end{tabular}


Table 1. Cont.

\begin{tabular}{|c|c|c|c|c|c|}
\hline GeneID & \multicolumn{2}{|c|}{ log2FoldChangePadj } & Annotation & Function & Reference \\
\hline DN50530_c5_g14 & 9.970503 & $3.83 \times 10^{-20}$ & $\begin{array}{l}\text { ZP domain-containing } \\
\text { protein-like }\end{array}$ & & \\
\hline DN46494_c0_g1 & 12.88190 & $4.01 \times 10^{-53}$ & $\begin{array}{l}\text { ZP domain-containing } \\
\text { protein-like }\end{array}$ & & \\
\hline DN64127_c3_g3 & 9.64628 & $4.61 \times 10^{-17}$ & $\begin{array}{l}\text { Embryonic poly }(A) \text {-binding } \\
\text { protein } A\end{array}$ & $\begin{array}{l}\text { Required for oocyte maturation and female } \\
\text { fertility. }\end{array}$ & [45] \\
\hline DN82911_c0_g1 & 8.20682 & $7.04 \times 10^{-6}$ & Insulinase & Destroys or inactivates insulin. Elevated & \\
\hline DN49876_c0_g1 & 8.44020 & $2.62 \times 10^{-7}$ & Insulinase & insulin promotes oogenesis and inhibits & {$[1,46]$} \\
\hline$D N 46576 \_c 0 \_g 1$ & 8.18360 & $9.56 \times 10^{-6}$ & G1/S-specific cyclin-E-like & immune responses. & \\
\hline DN49363_c0_g1 & 9.49947 & $1.08 \times 10^{-15}$ & G2/mitotic-specific cyclin- $A$ & & \\
\hline DN49147_c0_g1 & 11.21366 & $1.90 \times 10^{-33}$ & G2/mitotic-specific cyclin-B & & \\
\hline DN15801_c0_g1 & 9.55009 & $3.72 \times 10^{-16}$ & G2/mitotic-specific cyclin-B & $\begin{array}{l}\text { Eocyte maturation and the onset or } \\
\text { embryogenesis. }\end{array}$ & [47-49] \\
\hline DN47388_c2_g1 & 10.19335 & $2.76 \times 10^{-22}$ & G2/mitotic-specific cyclin-B-like & & \\
\hline DN49534_c0_g1 & 9.76583 & $2.58 \times 10^{-46}$ & $\begin{array}{l}\text { G2/mitotic-specific cyclin-B-like } \\
\text { isoform X4 }\end{array}$ & & \\
\hline DN8940_c0_g1 & 10.33337 & $1.07 \times 10^{-23}$ & $\begin{array}{l}\text { Cell division cycle protein } 20 \\
\text { homolog }\end{array}$ & & \\
\hline DN50119_c0_g2 & -6.06256 & $3.33 \times 10^{-13}$ & Apolipophorin III & Plays roles in lipid uptake by insect oocytes. & [50] \\
\hline DN71428_c0_g1 & -8.39452 & $1.39 \times 10^{-6}$ & Matrix metalloproteinase-16 & Some members of matrix metalloproteinase & \\
\hline DN56705_c2_g7 & -3.79775 & 0.01004 & Matrix metalloproteinase-24-like & $\begin{array}{l}\text { may play roles in corpus luteum formation, } \\
\text { follicular development, and ovulation. }\end{array}$ & [41] \\
\hline DN37027_c0_g1 & 11.90835 & $5.60 \times 10^{-42}$ & Zygote arrest protein 1 & $\begin{array}{l}\text { Ovary-specific maternal factor that plays } \\
\text { essential roles during the oocyte-to-embryo }\end{array}$ & [51] \\
\hline DN94405_c0_g1 & 12.16383 & $4.47 \times 10^{-45}$ & Zygote arrest protein 1 & transition. & \\
\hline DN84630_c0_g1 & 8.95337 & $5.28 \times 10^{-11}$ & Astacin-like; Ovastacin & $\begin{array}{l}\text { Cleaves ZP2 and prevents polyspermy; } \\
\text { degrades egg envelope and releases the } \\
\text { embryo from its intracapsular life. }\end{array}$ & [52] \\
\hline DN47329_c0_g1 & 10.45829 & $5.41 \times 10^{-25}$ & $\begin{array}{l}\text { Oocyte-specific histone RNA } \\
\text { stem-loop-binding protein } 2\end{array}$ & $\begin{array}{l}\text { Plays a role in the storage of } \\
\text { replication-dependent histone mRNAs and } \\
\text { proteins during oogenesis. }\end{array}$ & [53] \\
\hline
\end{tabular}

Table 2. Reproductive related KEGG pathways of DEGs in C. chuxiongica females.

\begin{tabular}{|c|c|c|c|c|c|}
\hline Pathway & Padj & $\begin{array}{c}\text { Number of } \\
\text { Genes }\end{array}$ & First Category & Second Category & Function \\
\hline \multicolumn{6}{|c|}{ Mated-1 h vs. Virgin-1 h: Up regulated DEGs } \\
\hline $\begin{array}{l}\text { Steroid hormone } \\
\text { biosynthesis }\end{array}$ & 0.03602 & 1 & Metabolism & Lipid metabolism & Egg maturation \\
\hline \multicolumn{6}{|c|}{ Mated-24 h vs. Virgin-24 h: Down regulated DEGs } \\
\hline $\begin{array}{l}\text { Estrogen signaling } \\
\text { pathway }\end{array}$ & 0.01301 & 4 & Organismal Systems & Endocrine system & Fecundity \\
\hline \multicolumn{6}{|c|}{ Mated-24 h vs. Virgin-24 h: Up regulated DEGs } \\
\hline Oocyte meiosis & 0.01082 & 18 & Cellular Processes & Cell growth and death & Egg maturation \\
\hline $\begin{array}{l}\text { Progesterone-mediated } \\
\text { oocyte maturation }\end{array}$ & 0.03177 & 13 & Organismal Systems & Endocrine system & Egg maturation \\
\hline
\end{tabular}


Table 3. Mating-induced expression changes in genes related to survivorship in C. chuxiongica females.

\begin{tabular}{|c|c|c|c|c|c|}
\hline GeneID & log2FoldChange & Padj & Annotation & Function & Reference \\
\hline \multicolumn{6}{|c|}{ Mated-1 h vs. Virgin-1 h } \\
\hline DN70573_c2_g1 & -5.42272 & $1.27 \times 10^{-9}$ & Defensin & Insect antimicrobial & \\
\hline DN60391_c5_g2 & -5.02886 & $2.84 \times 10^{-8}$ & Hymenoptaecin & peptides. & [54] \\
\hline \multicolumn{6}{|c|}{ Mated-6 h vs. Virgin- 6 h } \\
\hline DN63597_c1_g1 & -3.53279 & $\begin{array}{l}4.53 \times \\
10^{-12}\end{array}$ & Phenoloxidase & $\begin{array}{l}\text { Phenoloxidase involved in } \\
\text { defensive melanization } \\
\text { and production of } \\
\text { oxidative free radicals. }\end{array}$ & [1] \\
\hline \multicolumn{6}{|c|}{ Mated-24 h vs. Virgin-24 h } \\
\hline DN45974_c0_g1 & -6.77749 & $5.08 \times 10^{-7}$ & Megourin & $\begin{array}{l}\text { Insect antimicrobial } \\
\text { peptides. }\end{array}$ & [54] \\
\hline DN45254_c0_g1 & -6.34763 & $\begin{array}{l}4.15 \times \\
10^{-17}\end{array}$ & $\begin{array}{l}\text { Fungal protease } \\
\text { inhibitor-1-like }\end{array}$ & Against fungal infection. & {$[55]$} \\
\hline DN71273_c1_g5 & -2.95068 & 0.00896 & C-type lysozyme & $\begin{array}{l}\text { Defends against bacterial } \\
\text { infection by hydrolyzing } \\
\text { the bacterial cell walls and } \\
\text { causing bacterial lysis. }\end{array}$ & [56] \\
\hline DN51648_c2_g8 & -6.63010 & $\begin{array}{l}9.23 \times \\
10^{-11}\end{array}$ & $\begin{array}{c}\text { Heat shock protein } 27.2 \\
\text { (sHsp family) }\end{array}$ & & \\
\hline DN47336_c0_g1 & -5.01800 & $\begin{array}{l}1.63 \times \\
10^{-12}\end{array}$ & $\begin{array}{c}\text { Heat shock protein } 27.2 \\
\text { (sHsp family) }\end{array}$ & & \\
\hline DN71108_c1_g3 & -4.29401 & $\begin{array}{l}3.40 \times \\
10^{-11}\end{array}$ & $\begin{array}{c}\text { Heat shock protein } 27.2 \\
\text { (sHsp family) }\end{array}$ & & \\
\hline$D N 56298 \_c 0 \_g 1$ & -3.69216 & 0.02212 & $\begin{array}{c}\text { Heat shock protein } 27.2 \\
\text { (sHsp family) }\end{array}$ & $\begin{array}{l}\text { Heat shock and stress } \\
\text { response. }\end{array}$ & [4] \\
\hline DN70765_c7_g9 & -4.25919 & $\begin{array}{l}1.24 \times \\
10^{-13}\end{array}$ & $\begin{array}{l}\text { Heat shock protein } \\
\text { 70-like (Hsp70 family) }\end{array}$ & & \\
\hline DN57953_c3_g2 & -3.39554 & $\begin{array}{c}5.58 \times \\
10^{-10}\end{array}$ & $\begin{array}{l}\text { Heat shock protein } \\
\text { 70-like (Hsp70 family) }\end{array}$ & & \\
\hline DN62526_c5_g1 & -6.22937 & $1.06 \times 10^{-7}$ & $\begin{array}{l}\text { Heat shock protein } 70 \\
\text { cognate (Hsp70 family) }\end{array}$ & & \\
\hline$D N 62526 \_c 5 \_g 4$ & -4.59306 & 0.04497 & $\begin{array}{l}\text { Heat shock protein } 70 \\
\text { cognate (Hsp } 70 \text { family) }\end{array}$ & & \\
\hline DN67613_c0_g10 & 10.89725 & $\begin{array}{c}7.42 \times \\
10^{-30}\end{array}$ & $\begin{array}{l}\text { Heat shock protein } 70 \\
\text { (Hsp70 family) }\end{array}$ & & \\
\hline DN67613_c0_g5 & 8.479329 & $\begin{array}{l}6.81 \times \\
10^{-21}\end{array}$ & $\begin{array}{l}\text { Heat shock protein } 70 \\
\text { (Hsp70 family) }\end{array}$ & & \\
\hline DN67090_c3_g8 & 8.29444 & $2.51 \times 10^{-6}$ & $\begin{array}{l}\text { Heat shock protein } 70 \\
\text { domain containing } \\
\text { protein (Hsp70 family) }\end{array}$ & & \\
\hline$D N 64447 \_c 3 \_g 1$ & 7.39234 & 0.03894 & $\begin{array}{l}\text { Heat shock protein } 75 \\
\text { (Hsp90 family) }\end{array}$ & & \\
\hline DN64447_c4_g1 & 3.92852 & $\begin{array}{l}4.91 \times \\
10^{-11}\end{array}$ & $\begin{array}{l}\text { Heat shock protein } 90 \\
\text { (Hsp90 family) }\end{array}$ & & \\
\hline
\end{tabular}


Table 4. Survivorship related KEGG pathways enriched in DEGs of C. chuxiongica females.

\begin{tabular}{|c|c|c|c|c|c|}
\hline Pathway & Padj & $\begin{array}{l}\text { Number } \\
\text { of Genes }\end{array}$ & First Category & Second Category & Function \\
\hline \multicolumn{6}{|c|}{ Mated-1 h vs. Virgin-1 h: Down regulated DEGs } \\
\hline $\begin{array}{l}\text { Inflammatory bowel } \\
\text { disease (IBD) }\end{array}$ & 0.02764 & 1 & Human Diseases & Immune disease & Defense \\
\hline Legionellosis & 0.02981 & 1 & Human Diseases & Infectious disease: bacterial & Defense \\
\hline Salmonella infection & 0.03185 & 1 & Human Diseases & Infectious disease: bacterial & Defense \\
\hline $\begin{array}{c}\text { Pathogenic Escherichia coli } \\
\text { infection }\end{array}$ & 0.03341 & 1 & Human Diseases & Infectious disease: bacterial & Defense \\
\hline \multicolumn{6}{|c|}{ Mated-1 h vs. Virgin-1 h: Up regulated DEGs } \\
\hline $\begin{array}{c}\text { Inflammatory mediator } \\
\text { regulation of TRP } \\
\text { channels }\end{array}$ & 0.03394 & 1 & Organismal Systems & Sensory system & Defense \\
\hline $\begin{array}{l}\text { Complement and } \\
\text { coagulation cascades }\end{array}$ & 0.03397 & 1 & Organismal Systems & Immune system & Defense \\
\hline \multicolumn{6}{|c|}{ Mated-6 h vs. Virgin-6 h: Down regulated DEGs } \\
\hline $\begin{array}{l}\text { Complement and } \\
\text { coagulation cascades }\end{array}$ & 0.00061 & 5 & Organismal Systems & Immune system & Defense \\
\hline $\begin{array}{c}\text { Staphylococcus aureus } \\
\text { infection }\end{array}$ & 0.00791 & 3 & Human Diseases & Infectious disease: bacterial & Defense \\
\hline Platelet activation & 0.01418 & 4 & Organismal Systems & Immune system & Defense \\
\hline \multicolumn{6}{|c|}{ Mated-24 h vs. Virgin-24 h: Down regulated DEGs } \\
\hline $\begin{array}{l}\text { Antigen processing and } \\
\text { presentation }\end{array}$ & 0.00018 & 6 & Organismal Systems & Immune system & Defense \\
\hline Legionellosis & 0.00046 & 6 & Human Diseases & Infectious disease: bacterial & Defense \\
\hline Measles & 0.00319 & 5 & Human Diseases & Infectious disease: viral & Defense \\
\hline $\begin{array}{l}\text { Complement and } \\
\text { coagulation cascades }\end{array}$ & 0.00620 & 4 & Organismal Systems & Immune system & Defense \\
\hline Influenza A & 0.00987 & 5 & Human Diseases & Infectious disease: viral & Defense \\
\hline Toxoplasmosis & 0.01130 & 4 & Human Diseases & Infectious disease: parasitic & Defense \\
\hline Tuberculosis & 0.02795 & 4 & Human Diseases & Infectious disease: bacterial & Defense \\
\hline Amoebiasis & 0.02986 & 3 & Human Diseases & Infectious disease: parasitic & Defense \\
\hline $\begin{array}{l}\text { Staphylococcus aureus } \\
\text { infection }\end{array}$ & 0.04516 & 2 & Human Diseases & Infectious disease: bacterial & Defense \\
\hline $\begin{array}{l}\text { Longevity regulating } \\
\text { pathway - multiple species }\end{array}$ & 0.01139 & 4 & Organismal Systems & Aging & Aging \\
\hline \multicolumn{6}{|c|}{ Mated-24 h vs. Virgin-24 h: Up regulated DEGs } \\
\hline $\begin{array}{c}\text { Pathogenic Escherichia coli } \\
\text { infection }\end{array}$ & $6.25 \times 10^{-5}$ & 18 & Human Diseases & Infectious disease: bacterial & Defense \\
\hline Legionellosis & 0.01178 & 16 & Human Diseases & Infectious disease: bacterial & Defense \\
\hline Viral myocarditis & 0.02943 & 10 & Human Diseases & Cardiovascular disease & Defense \\
\hline
\end{tabular}

\subsection{Transcriptional Changes at $6 \mathrm{~h}$ Post-Mating}

Within the 320 DEGs of M6 h-vs.-V6 h, 206 were downregulated and 114 were upregulated in mated females compared to virgin ones (Figure 1b). The LC value of DEGs differed from -10.34 to 12.29 (Table S5).

Sixteen reproductive-related genes (all downregulated) were found within these DEGs (Table 1), including five Vg encoding genes (LC: -9.15 to -2.35 ), three Haemolymph juvenile hormone-binding protein-encoding genes (LC: -3.45 to -2.48 ), two Takeout-like protein encoding genes (LC: -2.34 to -1.59$)$, one UDP-glucosyltransferase like encoding gene (LC: -2.71 ), one matrix metalloproteinase encoding gene (LC: -8.16$)$, and four cytochrome P450 encoding genes (LC: -2.86 to -2.01 ). No reproductive-related KEGG pathways were significantly enriched to the 320 DEGs.

One immunity-related gene was found within these DEGs (Table 3), which is the phenoloxidase-encoding gene (LC: -3.53). Three immunity-related KEGG pathways were significantly enriched to 12 downregulated DEGs (Table 4). 
Still no longevity and heat shock related genes and pathways were found within these DEGs.

\subsection{Transcriptional Changes at $24 \mathrm{~h}$ Post-Mating}

Within the 1068 DEGs of M24 h-vs.-V24 h, 104 genes were downregulated and 964 genes were upregulated in mated females compared to virgin ones (Figure 1c). The LC value of DEGs changed from -10.50 to 14.64 (Table S6).

A total of 51 transcripts were annotated as reproductive related genes within these DEGs (Table 1), including seven Vitellogenin and Vitellogenin-like (all downregulated), one Vitellogenin-receptor (upregulated), 26 Zona pellucida protein-like (all upregulated), seven Cyclin-like (all upregulated), and ten other reproductive-related genes. Three reproductive related KEGG pathways were significantly enriched (Table 2): (1) Estrogen signaling pathway, enriched to four downregulated DEGs; (2) oocyte meiosis pathway, enriched to 18 upregulated DEGs; and (3) progesterone-mediated oocyte maturation pathway, enriched to 13 upregulated DEGs. To get some evolutional clues on oocyte maturation, unigenes annotated to the progesterone-mediated oocyte maturation pathway (Table S10) were mapped to the same pathway of Xenopus (KEGG id: map04914) (Figure 6). Results show about half of the genes on map04914 were found in the unigenes of C. chuxiongica. This study also revealed an additional 25 unmapped progesterone-mediated oocyte maturationrelated unigenes (Table S10).

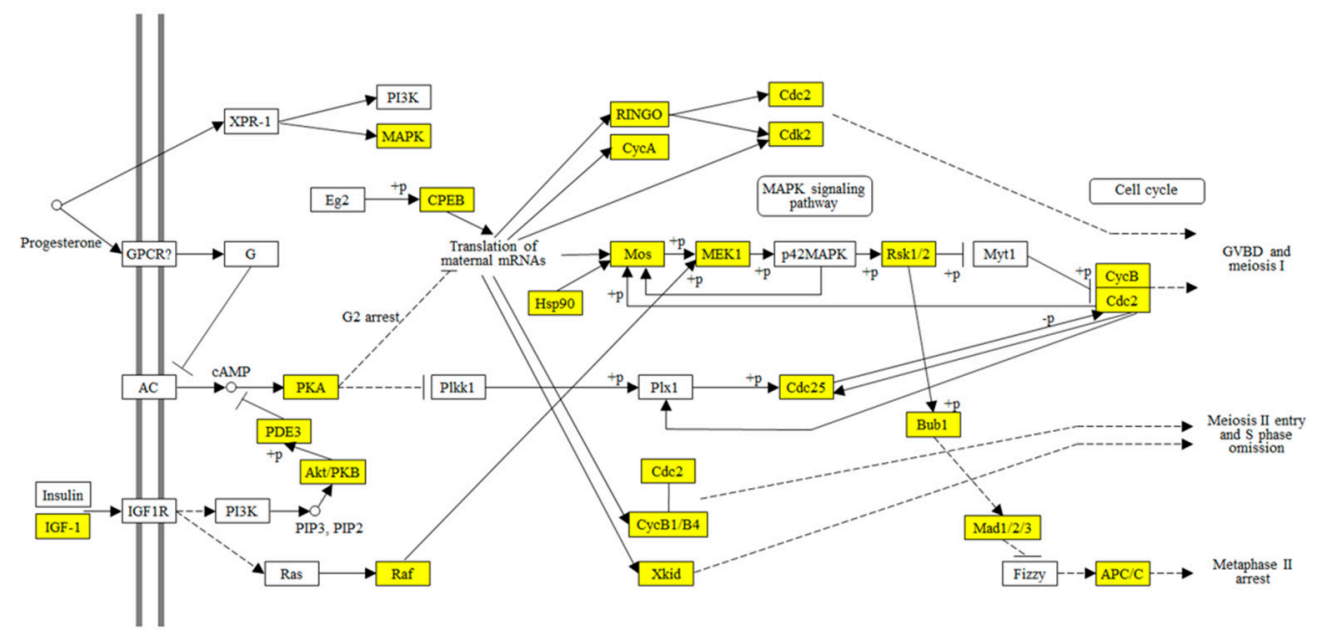

Figure 6. Mapping analysis of progesterone-mediated oocyte maturation pathway. Yellows represent the transcripts of $C$. chuxiongica females mapped to the Xenopus progesterone-mediated oocyte maturation pathway (KEGG id: map04914).

Three immunity related genes were found within these DEGs with all of them being downregulated (Table 3). Thirteen immunity-related KEGG pathways were significantly enriched, with ten of them being enriched to downregulated DEGs and three being enriched to upregulated DEGs (Table 4).

There are thirteen Hsps-encoding genes found within these DEGs (Table 3), including four sHsps (LC: -6.63 to -3.69 ), seven Hsp70s (LC: -6.23 to 10.90), and two Hsp90s (LC: 3.93 to 7.39 ).

One longevity-related pathway was significantly enriched, which was related to four downregulated DEGs (Table 4).

\subsection{Validation of RNAseq by $q R T-P C R$}

Eight DEGs from each group (M1 h-vs.-V1 h, M6 h-vs.-V6 h and M24 h-vs.-V24 h, respectively) were used to verify the accuracy of RNAseq, which include six reproductionrelated genes (DN64818_c0_g6, DN52313_c1_g5, DN65191_c2_g1,DN66978_c2_g1,DN78694_ $c 0 \_g 1$, and DN71364_c1_g1), three immunity-related genes (DN70573_c2_g1, DN60391_c5_g2 
and DN63597_c1_g1), and one heat shock response gene (DN70765_c7_g9) (Figure 7). The expression levels of these genes measured by qRT-PCR were similar to the results of RNAseq analysis, which suggested that the RNAseq data were reliable.
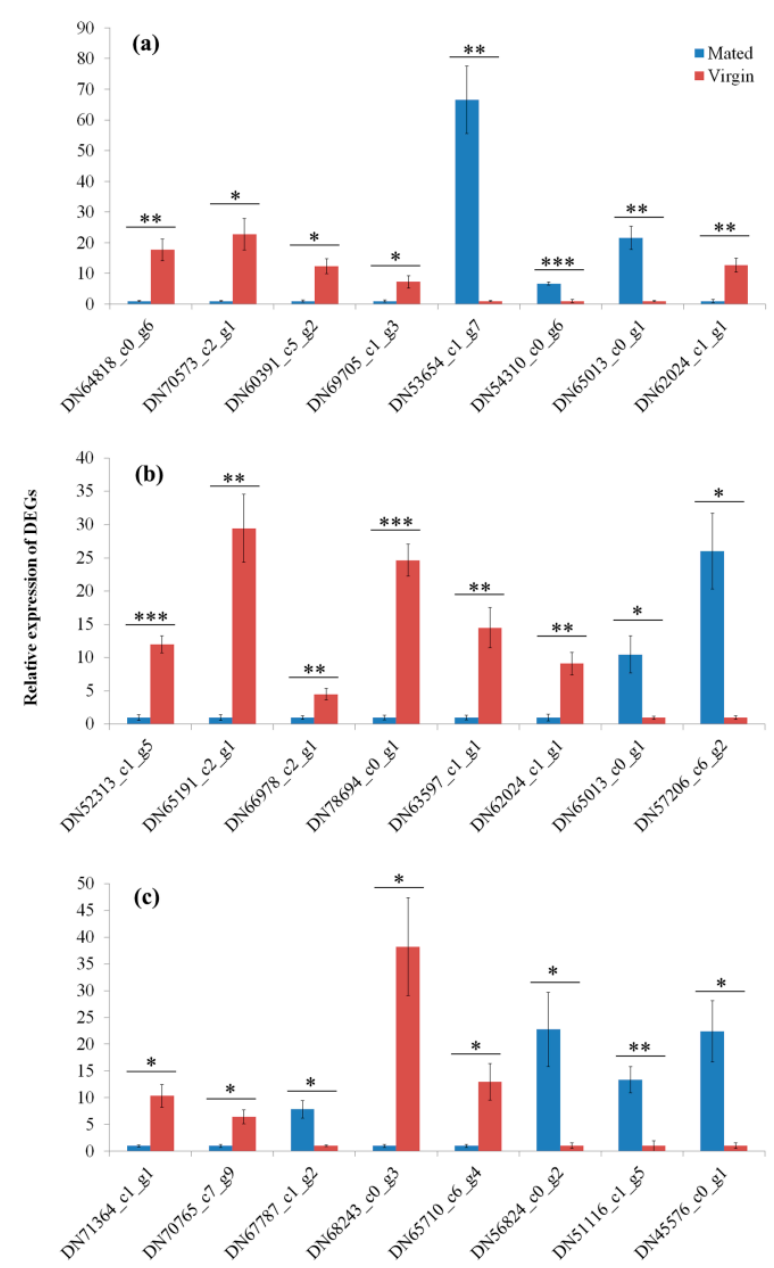

Figure 7. The validation of RNAseq results by quantitative real-time PCR. (a) DEGs from Mated-1 h vs. Virgin-1 h, (b) DEGs from Mated-6 h vs. Virgin-6 h, and (c) DEGs from Mated-24 h vs. Virgin-24 h. ${ }^{*}$ indicates $p<0.05 ;{ }^{* *}$ indicates $p<0.01,{ }^{* * *}$ indicates $p<0.001$. Error bars indicate SE.

\section{Discussion}

Previous studies generally found that mating induces the upregulation of egg production (fecundity)-related genes, such as YPs and Vg [15,18,19]. In the present study, however, we found an opposite result, where all Vg encoding DEGs (five in M6 h-vs.-V6 h and seven in M24 h-vs.-V24 h) were found downregulated largely (Table 1). Moreover, other positive modulator of fecundity, including Follicle cell protein, Haemolymph juvenile hormone binding protein, UDP-glucosyltransferase, Apolipophorin III, and Matrix metalloproteinase, were also downregulated significantly (Table 1). In addition, the upregulation of Insulinase (which destroys or inactivates insulin) in mated females may also negatively affect female fecundity. These results suggested that mating downregulated reproduction in terms of fecundity (number of eggs) in C. chuxiongica.

On the contrary, we found a significant upregulation on genes related to oocyte maturation and embryogenesis (Table 1): (1) Seven Cyclins, which play roles in the process of oocyte maturation and the onset of embryogenesis [47-49]; (2) two Zygote arrest protein, which are ovary-specific maternal factors that play essential roles during the oocyte-toembryo transition [51]. Moreover, two oocyte maturation-related pathways, the oocyte meiosis pathway and the progesterone-mediated oocyte maturation pathway, have been 
significantly enriched upregulated DEGs (Table 2). Fully grown oocytes are arrested in the first meiotic prophase (Figure 6), which is a state of low metabolic activity without detrimental effects on subsequent embryogenesis [57]. At the end step of oocyte maturation, a process termed meiotic reinitiation or resumption, takes place before or during the time when the oocyte moves from the follicle to the oviduct (ovulation). Meiotic maturation involves the activation of various signal transduction pathways, which converge to activate maturation-promoting factors (Figure 6). In vertebrate, the marks of meiotic maturation include [58]: (1) Resumption of meiosis I, including germinal vesicle breakdown, chromosome condensation, and spindle formation; (2) the transition from meiosis I to meiosis II; and (3) arrest in metaphase II. Meiosis II will complete after egg fertilization. In many invertebrates, however, oocytes maturation proceeds only to metaphase of meiosis I, which is when they are fertilized [58]. Therefore, the upregulation of genes and pathways in relation to oocyte meiosis and maturation in C. chuxiongica (Tables 1 and 2) are likely to function in meiotic resumption and maturation in fully grown oocytes, from which the egg is ready for the following fertilization.

The progesterone-mediated oocyte maturation pathway of the African clawed frog Xenopus laevis is the most intensively studied model system for meiotic maturation [58]. Therefore, we mapped unigenes that annotated to the progesterone-mediated oocyte maturation pathway to the same pathway of Xenopus (KEGG id: map04914) and found that about half of the genes on map04914 were present in the unigenes of $C$. chuxiongica (Figure 6; Table S10). Further searching found an additional 25 unmapped progesteronemediated oocyte maturation-related unigenes in C. chuxiongica (Table S10). These results suggest that this pathway may also play roles in egg maturation of $C$. chuxiongica but factors and signaling pathways may be species-specific. In the present study, we also found the presence of Cyclin-B1 (KO id: K05868) and Xkid (KO id: K10403; kinesin-like DNA-binding protein, also known as Kinesin-like protein, KIF22) [57] in the enriched DEGs (Figure 6, Table S10). These proteins are all dispensable for meiosis I entry but play essential roles in the progression from meiosis I to meiosis II [58]. As mentioned above, oocytes maturation in many invertebrates proceeds only to metaphase of meiosis I until they are fertilized. If it is the case in C. chuxiongica, then the upregulation of Cyclin-B1 and Xkid may be triggered by mating factors (such as male accessary protein and sperms) or fertilization, which then will promote the transition of eggs from meiosis I to meiosis II. This warrants further studies.

More interestingly, this study also found significant expression changes in a number of genes that may relate to fertilization and egg hatching, including a number of Zona pellucida protein and Ovastacin/Astacin-like (Table 1). The Zona pellucida (ZP) is a glycoprotein layer surrounding the plasma membrane of mammalian oocytes, which is a vital constitutive part of the oocyte and functions in primary binding and induction of the sperm acrosome reaction [42,44]. A family of $18 \mathrm{ZP}$-like protein encoding genes has been identified in $D$. melanogaster [59]. These genes are specifically expressed during embryogenesis and during differentiation of epithelial tissues. However, whether these ZP-like proteins also play roles in fertilization is still unknown in $D$. melanogaster or other insect species because little is known about the molecular mechanism of fertilization in insects [59]. Ovastacin plays a role in the post-fertilization block to sperm binding by cleaving ZP2 in the ZP to prevent polyspermy in mouse [52]. Some astacins may function as hatching enzyme in insects, which degrades the egg envelope to release the embryo [60]. The reproductiverelated regulation on these genes may suggest they are crucial in the reproduction process of insects.

Insect cytochrome P450 families contain a class of enzymes, which play diverse functions in detoxification and the biosynthesis of hormones [34]. In Drosophila, mating induced downregulation in six P450 genes and upregulation in $22 \mathrm{P} 450$ genes. In B. tabaci, mating induced upregulation in two P450 genes [20]. In the present study, one P450 gene (CYP2C) was significantly upregulated in mated females shortly after mating ( $1 \mathrm{~h}$ postmating) and four P450 genes were downregulated in mated females at $6 \mathrm{~h}$ post-mating 
(Table 1). Upregulation of P450 shortly after mating may be a detoxification response as males may transfer slightly toxic seminal fluid during mating [61] and downregulation of P450 sometime after mating may be due to trade-offs between reproduction and survival.

Reproduction associated heat shock responses are inconsistent in different species, which is downregulated after the onset of reproduction in C. elegans [4] while in honey bees, increased reproduction did not cause loss of heat shock response in the reproductive queen [3]. In B. tabaci, mating led to the upregulation of Hsp68 and Hsp70 genes at different time points in mated females [20]. In the present study, we also found significant expression changes in thirteen Hsps-encoding transcripts $24 \mathrm{~h}$ post-mating, with some of them (four sHsps and four Hsp70s) being downregulated and others (three Hsp70s and two Hsp90s) being upregulated substantially (Table 3). Hsps act as molecular chaperones to improve organisms' survival, development, and reproduction under different stresses. Studies have shown that Hsps have multiple functions during reproductive process, including gamete protection [58], oocyte maturation (Figure 6) [62], reproduction vs. survival trade-offs [63], etc. In addition, one longevity-associated pathway was significantly enriched, which related to four downregulated Hsp70s coding DEGs (Table 4). Therefore, mating induced different response in different Hsps may be due to their different functions in reproduction and survival.

Insect immune defenses are carried out through humoral and hemocyte responses, with the former playing defensive role through the synthesis of antimicrobial peptides and the latter through encapsulation and phagocytosis [64]. In the present study, four antimicrobial peptides encoding gene (Defensin, Hymenoptaecin, Megourin, and Fungal protease inhibitor) were found in C. chuxiongica and all of them were downregulated considerably in mated females (Table 3). In addition, two hemocyte responses related enzyme-encoding genes, Phenoloxidase and Lysozyme, were also found downregulated in mated females (Table 3). Lysozyme is involved in immune defense by hydrolyzing the bacterial cell walls whereas phenoloxidase participates in the synthesis of oxidative free radicals and the defensive melanization [1]. A total of 21 immunity related KEGG pathways were enriched, with sixteen of them being enriched to downregulated DEGs and five being enriched to upregulated DEGs. These results have suggested that the immunity activity has been downregulated substantially in mated females.

Above results and discussion have suggested that mating is also an essential switch in C. chuxiongica, a species with facultative parthenogenesis, which induced significant trade-offs between reproduction and survivorship. As mentioned above, the male and female adults of $C$. chuxiongica can mate a few hours after eclosion and mated females start to lay fertilized eggs in the subsequent day after mating, as well as start to show egg protection behavior; unmated females (virgin) may start to lay unfertilized eggs through parthenogenesis a few days ( $>3 \mathrm{~d}$ ) after eclosion [21]. Therefore, the pattern of gene expression changes after mating is consistent with the post-mating behavioral and physiological changes. Moreover, the present study also suggested that mating may also induce trade-offs within reproduction on fecundity vs. fertility in C. chuxiongica females, in which females may downregulate oogenesis and egg production to restrict fecundity but upregulate meiotic maturation in fully grown oocytes and fertilization-related pathways to favor the following egg fertilization. C. chuxiongica mostly occurs in pine forests at high altitude and barren soil, its larvae are obligate pine defoliator, which mainly feed on Pinus yunnanensis [21]. C. chuxiongica has a long (19 months) larval dormancy stage and adults do not feed [21], which may force adults to use limited resources optimally on reproduction and survival. In addition, C. chuxiongica females lay eggs on the surface of pine needles [23], which will facilitate larval feeding after hatching but also will likely to incur attack by natural enemies. C. chuxiongica females may thus have evolved egg protection behavior. The fecundity of C. chuxiongica is about 50 eggs per female [22]. Therefore, compared to other high fecundity insect species, such as S. litura that lay more than one thousand eggs per female [65] and show upregulation of fecundity related genes after mating [15], C. chuxiongica may have evolved a different reproductive strategy, i.e., 
restrict whole fecundity while ensuring higher egg fertilization and offspring survival. Future studies to clarify the hypotheses established in this study by using other techniques, such as RNAi and 2D electrophoresis/MS, will help to provide deeper insights in this field.

The present study and previous studies [21-23] on C. chuxiongica suggest that this species has evolved multiple physiological and behavioral strategies (such as facultative parthenogenesis, long larval diapause, and trade-offs on reproduction and survival) to adapt to its living environment.

Supplementary Materials: The following are available online at https: / www.mdpi.com/article / 10.3390/insects12080693/s1. Table S1 Primers for quantitative real-time PCR. Table S2 Summary of the quality of all sample sequencing data. Table S3 Pearson's correlation coefficient. Table S4 Differentially expressed genes (DEGs) between M1 h-vs.-V1 h group. Table S5 Differentially expressed genes (DEGs) between M6 h-vs.-V6 h group. Table S6 Differentially expressed genes (DEGs) between M24 h-vs.-V24 h group. Table S7 Identical DEGs between groups. Table S8 GO enrichment of DEGs from different groups. Table S9 KEGG enrichment of DEGs from different groups. Table S10 Progesterone-mediated oocyte maturation related unigenes.

Author Contributions: Conceptualization, J.X., J.-H.L., H.Y. and P.C.; methodology, H.Y., M.-R.S. and P.C.; investigation, H.Y., M.-R.S., J.X. and J.-H.L.; data analysis, H.Y., M.-R.S. and J.X.; writing, H.Y., J.X. and J.-H.L. All authors have read and agreed to the published version of the manuscript.

Funding: The research reported here was supported by projects from the Joint Special Project of Yunnan Province for Basic Agricultural Research (2018FG001-002), the Science and Technology Planning Project in Key Areas of Yunnan Province (202001BB050002) and the National Natural Science Foundation Program of China (32060357; 31760635).

Institutional Review Board Statement: Not applicable.

Data Availability Statement: The transcriptome raw reads have been deposited to the NCBI SRA database (accession no.: SRR13991392 SRR13991407). Other data generated or analyzed during this study were included in this article and its Supplementary Information.

Acknowledgments: We thank Su Ping Ong from Forest Research Institute Malaysia (FRIM) for English improvements, and the editor and reviewers for their constructive comments.

Conflicts of Interest: The authors declare no conflict of interest.

\section{References}

1. Schwenke, R.A.; Lazzaro, B.P.; Wolfner, M.F. Reproduction-immunity trade-offs in insects. Annu. Rev. Entomol. 2016, 61, 239-256. [CrossRef] [PubMed]

2. Rodrigues, M.A.; Flatt, T. Endocrine uncoupling of the trade-off between reproduction and somatic maintenance in eusocial insects. Curr. Opin. Insect Sci. 2016, 16, 1-8. [CrossRef] [PubMed]

3. Shih, S.R.; Huntsman, E.M.; Flores, M.E.; Snow, J.W. Reproductive potential does not cause loss of heat shock response performance in honey bees. Sci. Rep. 2020, 10, 19610. [CrossRef]

4. Labbadia, J.; Morimoto, R.I. Repression of the heat shock response is a programmed event at the onset of reproduction. Mol. Cell 2015, 59, 639-650. [CrossRef]

5. Oku, K.; Price, T.A.R.; Wedell, N. Does mating negatively affect female immune defences in insects? Anim. Biol. 2019, 69, 117-136. [CrossRef]

6. Stearns, S.C. The Evolution of Life Histories; Oxford University Press: Oxford, UK, 1992.

7. Flatt, T. Survival costs of reproduction in Drosophila. Exp. Gerontol. 2011, 46, 369-375. [CrossRef] [PubMed]

8. Flatt, T.; Heyland, A.; Rus, F.; Porpiglia, E.; Sherlock, C.; Yamamoto, R.; Garbuzov, A.; Palli, S.R.; Tatar, M.; Silverman, N. Hormonal regulation of the humoral innate immune response in Drosophila melanogaster. J. Exp. Biol. 2008, 211, $2712-2724$. [CrossRef] [PubMed]

9. Zhang, Z.; Palli, S.R. Identification of a cis-regulatory element required for 20-hydroxyecdysone enhancement of antimicrobial peptide gene expression in Drosophila melanogaster. Insect Mol. Biol. 2009, 18, 595-605. [CrossRef] [PubMed]

10. Baker, H.G.; Baker, I. Amino-acids in nectar and their evolutionary significance. Nature 1973, 241, 543-545. [CrossRef]

11. Surendra, K.C.; Olivier, R.; Tomberlin, J.K.; Jha, R.; Khanal, S.K. Bioconversion of organic wastes into biodiesel and animal feed via insect farming. Renew. Energy 2016, 98, 197-202. [CrossRef]

12. Soller, M.; Bownes, M.; Kubli, E. Mating and sex peptide stimulate the accumulation of yolk in oocytes of Drosophila melanogaster. Eur. J. Biochem. 1997, 243, 732-738. [CrossRef] [PubMed] 
13. Yu, J.F.; Li, C.; Xu, J.; Liu, J.H.; Ye, H. Male accessory gland secretions modulate female post-mating behavior in the moth Spodoptera litura. J. Insect. Behav. 2014, 27, 105-116. [CrossRef]

14. Yapici, N.; Kim, Y.J.; Ribeiro, C.; Dickson, B.J. A receptor that mediates the post-mating switch in Drosophila reproductive behaviour. Nature 2008, 451, 33-37. [CrossRef] [PubMed]

15. Gao, B.; Song, X.Q.; Yu, H.; Fu, D.Y.; Xu, J.; Ye, H. Mating-induced differential expression in genes related to reproduction and immunity in Spodoptera litura (Lepidoptera: Noctuidae) female moths. J. Insect Sci. 2020, 20, 10. [CrossRef]

16. Wigby, S.; Suarez, S.S.; Lazzaro, B.P.; Pizzari, T.; Wolfner, M.F. Sperm success and immunity. Curr. Top. Dev. Biol. 2019, 135, 287-313.

17. Okada, K.; Suzaki, Y.; Sasaki, R.; Katsuki, M. Fitness costs of polyandry to female cigarette beetle Lasioderma serricorne. Behav. Ecol. Sociobiol. 2017, 71, 86. [CrossRef]

18. Zhou, S.; Mackay, T.F.C.; Anholt, R.R.H. Transcriptional and epigenetic responses to mating and aging in Drosophila melanogaster. BMC Genom. 2014, 15, 927. [CrossRef]

19. Dalton, J.E.; Kacheria, T.S.; Knott, S.R.V.; Lebo, M.S.; Nishitani, A.; Sanders, L.E.; Stirling, E.J.; Winbush, A.; Arbeitman, M.N. Dynamic, mating-induced gene expression changes in female head and brain tissues of Drosophila melanogaster. BMC Genom. 2010, 11, 541. [CrossRef] [PubMed]

20. Huo, Z.; Liu, Y.; Yang, J.; Xie, W.; Wang, S.; Wu, Q.; Zhou, X.; Pang, B.; Zhang, Y. Transcriptomic analysis of mating responses in Bemisia tabaci MED females. Insects 2020, 11, 308. [CrossRef]

21. Li, Y.-H.; Xu, J. Geographical Distribution, Biological Characteristics and Diapause Mechanism of Cephalica Chuxiongica; China Forestry Publishing House: Beijing, China, 2020; p. 226.

22. Fan, J.-X. Study on Biological Characteristics and Control of Cephalcia Chuxiongica Xiao; Southwest Forestry University: Kunming, China, 2017.

23. Yu, H.; Du, C.-M.; Shi, M.-R.; Feng, L.; Fu, D.-Y.; Xu, J.; Li, Y.-H. The diversity and function of intestinal microorganisms in four geographic Cephalcia chuxiongica (a pine defoliator) populations. J. Appl. Entomol. 2021, 145, 394-405. [CrossRef]

24. Taeger, A.; Blank, S.M.; Liston, A.D. World catalog of Symphyta (Hymenoptera). Zootaxa 2010, 2580, 3-1064. [CrossRef]

25. Zhang, N. A Systematic Study of Cephalcia (Hymenoptera: Pamphiliidae) from China; Central South University of Forestry and Technology: Changsha, China, 2019.

26. Baine, Q.; Looney, C.; Smith, D.R.; Schiff, N.M.; Goulet, H.; Redford, A.J. About Sawflies. Available online: https://idtools.org/ id/sawfly/sawflies_biology.php (accessed on 5 March 2021).

27. Yan, Z.-L.; Ma, H.-F.; Mao, Y.-L.; Liu, L. Gravid females of Cephalcia chuxiongica (Hymenoptera, Pamphiliidae) are attracted to egg-carrying needles of Pinus yunnanensis. J. Hymenopt. Res. 2018, 65, 157-166. [CrossRef]

28. Grabherr, M.G.; Haas, B.J.; Yassour, M.; Levin, J.Z.; Thompson, D.A.; Amit, I.; Adiconis, X.; Fan, L.; Raychowdhury, R.; Zeng, Q.; et al. Full-length transcriptome assembly from RNA-Seq data without a reference genome. Nat. Biotechnol. 2011, $29,644-652$. [CrossRef]

29. Pertea, G.; Huang, X.; Liang, F.; Antonescu, V.; Sultana, R.; Karamycheva, S.; Lee, Y.; White, J.; Cheung, F.; Parvizi, B.; et al. TIGR gene indices clustering tools (TGICL): A software system for fast clustering of large EST datasets. Bioinformatics 2003, 19, 651-652. [CrossRef]

30. Storey, J.D. The positive false discovery rate: A Bayesian interpretation and the q-value. Ann. Stat. 2003, 31, 2013-2035. [CrossRef]

31. Altschul, S.F.; Madden, T.L.; Schaffer, A.A.; Zhang, J.; Zhang, Z.; Miller, W.; Lipman, D.J. Gapped BLAST and PSI-BLAST: A new generation of protein database search programs. Nucleic Acids Res. 1997, 25, 3389-3402. [CrossRef] [PubMed]

32. Livak, K.J.; Schmittgen, T.D. Analysis of relative gene expression data using real-time quantitative PCR and the $2^{-\Delta \Delta C T}$ method. Methods 2001, 25, 402-408. [CrossRef] [PubMed]

33. Burke, T.; Waring, G.L.; Popodi, E.; Minoo, P. Characterization and sequence of follicle cell genes selectively expressed during vitelline membrane formation in Drosophila. Dev. Biol. 1987, 124, 441-450. [CrossRef]

34. Feyereisen, R. Insect CYP genes and P450 enzymes. In Insect Molecular Biology and Biochemistry; Gilbert, L.I., Ed.; Academic Press: San Diego, CA, USA, 2012; pp. 236-316.

35. Upadhyay, S.K.; Singh, H.; Dixit, S.; Mendu, V.; Verma, P.C. Molecular characterization of vitellogenin and vitellogenin receptor of Bemisia tabaci. PLoS ONE 2016, 11, e0155306.

36. Van Rompay, L.; Borghgraef, C.; Beets, I.; Caers, J.; Temmerman, L. New genetic regulators question relevance of abundant yolk protein production in C. elegans. Sci. Rep. 2015, 5, 16381. [CrossRef] [PubMed]

37. Audsley, N.; Matthews, H.J.; Price, N.R.; Weaver, R.J. Allatoregulatory peptides in Lepidoptera, structures, distribution and functions. J. Insect Physiol. 2008, 54, 969-980. [CrossRef] [PubMed]

38. Pietrzyk, A.J.; Jaskolski, M.; Bujacz, G. Structural studies of juvenile hormone binding proteins. In Juvenile Hormones and Juvenoids: Modeling Biological Effects and Environmental Fate; Devillers, J., Ed.; CRC Press-Taylor \& Francis Group: Boca Raton, FL, USA, 2013; pp. 291-309.

39. Saurabh, S.; Vanaphan, N.; Wen, W.; Dauwalder, B. High functional conservation of takeout family members in a courtship model system. PLoS ONE 2018, 13, e0204615. [CrossRef]

40. Ge, L.Q.; Zheng, S.; Gu, H.T.; Zhou, Y.K.; Zhou, Z.; Song, Q.S.; Stanley, D. Jinggangmycin-induced UDP-glycosyltransferase 1-2-like is a positive modulator of fecundity and population growth in Nilaparvata lugens (Stal) (Hemiptera: Delphacidae). Front. Physiol. 2019, 10, 747. [CrossRef] [PubMed] 
41. Deady, L.D.; Shen, W.; Mosure, S.A.; Spradling, A.C.; Sun, J. Matrix metalloproteinase 2 is required for ovulation and corpus luteum formation in Drosophila. Plos Genet. 2015, 11, e1004989. [CrossRef]

42. Saldivar-Hernandez, A.; Gonzalez-Gonzalez, M.E.; Sanchez-Tusie, A.; Maldonado-Rosas, I.; Lopez, P.; Trevino, C.L.; Larrea, F.; Chirinos, M. Human sperm degradation of zona pellucida proteins contributes to fertilization. Reprod. Biol. Endocrinol. 2015, 13, 99. [CrossRef]

43. Gupta, S.K.; Bansal, P.; Ganguly, A.; Bhandari, B.; Chakrabarti, K. Human zona pellucida glycoproteins: Functional relevance during fertilization. J. Reprod. Immunol. 2009, 83, 50-55. [CrossRef] [PubMed]

44. Gupta, S.K.; Bhandari, B.; Shrestha, A.; Biswal, B.K.; Palaniappan, C.; Malhotra, S.S.; Gupta, N. Mammalian zona pellucida glycoproteins: Structure and function during fertilization. Cell Tissue Res. 2012, 349, 665-678. [CrossRef] [PubMed]

45. Guzeloglu-Kayisli, O.; Lalioti, M.D.; Aydiner, F.; Sasson, I.; Ilbay, O.; Sakkas, D.; Lowther, K.M.; Mehlmann, L.M.; Seli, E. Embryonic poly(A)-binding protein (EPAB) is required for oocyte maturation and female fertility in mice. Biochem. J. 2012, 446, 47-58. [CrossRef] [PubMed]

46. Galagovsky, D.; Katz, M.J.; Acevedo, J.M.; Sorianello, E.; Glavic, A.; Wappner, P. The Drosophila insulin-degrading enzyme restricts growth by modulating the PI3K pathway in a cell-autonomous manner. Mol. Biol. Cell 2014, 25, 916-924. [CrossRef]

47. Bourouh, M.; Dhaliwal, R.; Rana, K.; Sinha, S.; Guo, Z.; Swan, A. Distinct and overlapping requirements for Cyclins A, B, and B3 in Drosophila female meiosis. G3 Genes Genomes Genet. 2016, 6, 3711-3724. [CrossRef]

48. McCleland, M.L.; Farrell, J.A.; O'Farrell, P.H. Influence of cyclin type and dose on mitotic entry and progression in the early Drosophila embryo. J. Cell Biol. 2009, 184, 639-646. [CrossRef] [PubMed]

49. Vardy, L.; Pesin, J.A.; Orr-Weaver, T.L. Regulation of Cyclin A protein in meiosis and early embryogenesis. Proc. Natl. Acad. Sci. USA 2009, 106, 1838-1843. [CrossRef] [PubMed]

50. Ziegler, R.; Van Antwerpen, R. Lipid uptake by insect oocytes. Insect Biochem. Molec. 2006, 36, 264-272. [CrossRef] [PubMed]

51. Wu, X.M.; Wang, P.; Brown, C.A.; Zilinski, C.A.; Matzuk, M.M. Zygote arrest 1 (Zar1) is an evolutionarily conserved gene expressed in vertebrate ovaries. Biol. Reprod. 2003, 69, 861-867. [CrossRef]

52. Burkart, A.D.; Xiong, B.; Baibakov, B.; Jimenez-Movilla, M.; Dean, J. Ovastacin, a cortical granule protease, cleaves ZP2 in the zona pellucida to prevent polyspermy. J. Cell Biol. 2012, 197, 37-44. [CrossRef]

53. He, W.X.; Wu, M.; Liu, Z.; Li, Z.; Wang, Y.; Zhou, J.; Yu, P.; Zhang, X.J.; Zhou, L.; Gui, J.F. Oocyte-specific maternal Slbp2 is required for replication-dependent histone storage and early nuclear cleavage in zebrafish oogenesis and embryogenesis. $R N A$ 2018, 24, 1738-1748. [CrossRef]

54. Wu, Q.; Patocka, J.; Kuca, K. Insect antimicrobial peptides, a mini review. Toxins 2018, 10, 461. [CrossRef]

55. Roy, S.; Aravind, P.; Madhurantakam, C.; Ghosh, A.K.; Sankaranarayanan, R.; Das, A.K. Crystal structure of a fungal protease inhibitor from Antheraea mylitta. J. Struct. Biol. 2009, 166, 79-87. [CrossRef]

56. Ragan, E.J.; An, C.; Jiang, H.; Kanost, M.R. Roles of haemolymph proteins in antimicrobial defences of Manduca sexta. In Insect Infection and Immunity: Evolution, Ecology, and Mechanisms; Rolff, J., Reynolds, S.E., Eds.; Oxford University Press: Oxford, UK, 2009; pp. 34-48.

57. Perez, L.H.; Antonio, C.; Flament, S.; Vernos, I.; Nebreda, A.R. Xkid chromokinesin is required for the meiosis I to meiosis II transition in Xenopus laevis oocytes. Nat. Cell Biol. 2002, 4, 737-742. [CrossRef]

58. Schmitt, A.; Nebreda, A.R. Signalling pathways in oocyte meiotic maturation. J. Cell Sci. 2002, 115, 2457-2459. [CrossRef]

59. Litscher, E.S.; Wassarman, P.M. A Guide to Zona Pellucida Domain Proteins; John Wiley \& Sons: Hoboken, NJ, USA, 2015.

60. Lu, F.-K.; Tang, S.-M.; Shen, X.-J.; Wang, N.; Zhao, Q.-L.; Zhang, G.-Z.; Guo, X.-J. Molecular cloning and characterization of hatching enzyme-like gene in the silkworm, Bombyx mori. Mol. Biol. Rep. 2010, 37, 1175-1182. [CrossRef] [PubMed]

61. Innocenti, P.; Morrow, E.H. Immunogenic males: A genome-wide analysis of reproduction and the cost of mating in Drosophila melanogaster females. J. Evol. Biol. 2009, 22, 964-973. [CrossRef] [PubMed]

62. Will, T.; Schmidtberg, H.; Skaljac, M.; Vilcinskas, A. Heat shock protein 83 plays pleiotropic roles in embryogenesis, longevity, and fecundity of the pea aphid Acyrthosiphon pisum. Dev. Genes. Evol. 2017, 227, 1-9. [CrossRef] [PubMed]

63. Okada, Y.; Teramura, K.; Takahashi, K.H. Heat shock proteins mediate trade-offs between early-life reproduction and late survival in Drosophila melanogaster. Physiol. Entomol. 2014, 39, 304-312. [CrossRef]

64. Jiang, H.; Vilcinskas, A.; Kanost, M.R. Immunity in lepidopteran insects. In Invertebrate Immunity; Soderhall, K., Ed.; Springer: Boston, MA, USA, 2010; Volume 708, pp. 181-204.

65. $\mathrm{Xu}, \mathrm{J} . ;$ Wang, Q. Seminal fluid reduces female longevity and stimulates egg production and sperm trigger oviposition in a moth. J. Insect Physiol. 2011, 57, 385-390. [CrossRef] [PubMed] 